\title{
Optimal Controller Design for the System of Ball-on-sphere: The Linear Quadratic Gaussian (LQG) Case
}

\author{
Usman Mohammed \\ Department of Elect/Elect Engineering, Nile University of Nigeria, Abuja, Nigeria \\ Email: usman070064@nileuniversity.edu.ng

\section{Tologon Karataev} \\ Department of Elect/Elect Engineering, Nile University of Nigeria, Abuja, Nigeria \\ Email: tologon.karataev@nileuniversity.edu.ng \\ Omotayo O. Oshiga \\ Department of Elect/Elect Engineering, Nile University of Nigeria, Abuja, Nigeria \\ Email: ooshiga@ nileuniversity.edu.ng \\ Suleiman U. Hussein \\ Department of Elect/Elect Engineering, Nile University of Nigeria, Abuja, Nigeria \\ Email: elsuligh@gmail.com
}

Received: 12 February 2021; Accepted: 01 March 2021; Published: 08 April 2021

\begin{abstract}
Control system plays a critical function as one of the essential bedrocks of contemporary social development. Differential equations are time-based equations. The analysis of these equations according to time-domain, is what the theory of modern control is based on. It uses a state-space method which allows direct design in the time-domain. With the state-space method, many controllers can be designed optimally. LQG is one of these controllers. This controller is covered much in the literature. Despite this, not many works cover the ball-on-sphere system. Therefore, the research designed an optimal LQG controller for the system of ball-on-sphere. System dynamics were first investigated and the mathematical model was derived. After that, the system was linearized and then the state-space representation was obtained. Using this representation, the controller was designed and applied to the system for control. The control was done based on the specified desired system performance. Finally, the controller's performance was analyzed. Results obtained showed that the controller met the desired system performance. The controller satisfied the at least $80 \%$ performance requirement with $\theta_{x}$ is $82.35 \%$ and $\theta_{y}$ is $82.95 \%$ less than their respective unregulated settling times. It was also observed that minimizing the total control energy leads to maximizing the total transient energy. Another finding was that all states played role in regulating the controller to the desired system performance. Unfortunately, a settling time (of the ball's angles) of less than $1.00 \mathrm{sec}$ could not be realized. The realized performance is $2.35 \%$ and 2.95\% more than the desired performance in $x$ and $y$ directions, respectively, for the ball's angles settling time. This research is significant because it is the first to design an LQG controller for the ball-on-sphere system. Therefore, bridging the existing gap in the literature is the value of this research.
\end{abstract}

Index Terms: Optimality; Controller; Ball-on-sphere; Gaussian; System

\section{Introduction}

Control media, control subject, and controlled object are components that made up a control system. Control system's knowledge can be applied in many ways. For example, variables in devices, machines, mechanisms, etc., can be changed or maintain by applying this knowledge. This means that, with the application of different control schemes, the devices that are being controlled could be made to attain a specified preordained ideal state [1]. Control system plays a critical function as one of the essential bedrocks of contemporary social development. Therefore, there exists a direct correlation between humans' daily lives and control systems, which means that many applications in humans' daily life cannot be separated from control systems. These applications include; automated aircraft landings, 
temperature adjustment for conditioning air, changing the lifting speed of an elevator, etc. Conversely, control systems are not restricted to only humans' daily lives. They are commonly used in industry and, of course, scientific research [2]. The analysis of stability and dynamics of a system that is made up of an object and a ball on it has been often attempted in recent times for control purposes. These kinds of systems are widely used in the control engineering field, as well as in education, for research and industrial purposes. They include ball-on-sphere [3], ball and beam [4], ball-on-wheel [5], etc.

A laboratory experiment, that is web-based and can be accessed, in real-time, remotely, for controlling the system of ball-on-sphere is suggested in [6]. The system comprises a ball, 2 friction wheels, a sphere, and 2 motors. The motors drive the friction wheels while the friction wheels control the sphere's rolling along the 2 horizontal axes.

So, balancing the ball on the sphere is the main objective of the control experiment and this balancing is achieved by controlling the sphere's rolling. Due to its under-actuated, nonlinear, as well as, unstable nature, it is not an easy task to control this kind of system. Understanding the fundamental principles of control theory and nonlinear dynamics is very important. So, from an educational perspective, this system is appropriate for illustrations in class and experiments in a control laboratory to plainly illustrate and underline these principles. There are systems whereby nonlinearity affects the controller or the plant. These systems are called nonlinear control systems [7]. The majority of physical systems are naturally nonlinear. Since it is difficult to analyze nonlinear systems, linearizing them about their equilibrium points to get a linear system, and use the linear model for analysis is the usual practice in engineering. The system of ball-on-sphere is nonlinear, so it can be linearized about its equilibrium point as per the conventional practice for simplicity of analysis and control. An equilibrium point is a point where all variables, input, and state, are zero [5]. For control design purposes, the exact dynamic is extremely difficult to analyze [8]. So, the system of ball-on-sphere is reduced to 2 separated systems of ball-and-wheel, by ignoring the coupling terms that are of a higher order, whereby the control of the wheel's angle balances the ball over the wheel's periphery [5].

The control engineering field has a lot of fascinating and difficult facets. One of the most difficult facets of this field is balancing. There are many control systems that balancing is used as a control scheme such as the double inverted pendulum, system of ball-on-sphere, inverted pendulum, system of ball and beam, etc. [9]. Linear Quadratic Gaussian (LQG) controller is one of the most popular control schemes to balance these systems.

Because noise, assumed to be Gaussian, is principally taking into consideration in the output equations as well as the state equations, the LQG method is considered to be a robust control method. Moreover, in the design of the controller, the noise quantitative information is used. The LQG method is based on the principle called the separation principle.

According to this principle, the optimal control, which is Linear Quadratic Regulator (LQR), and the optimal estimation, which is the Kalman filter, are solved independently.

This means that the Kalman filter is designed first, which gives estimated states, then these estimated states are used to design the LQR controller as if they are exactly measurable [10]. The LQG control also uses the state-space representation of systems for controller design. In the absence of the state-space representation, LQG control needs to do identification of the system based on the provided input/output data. But when there available the state-space representation of a system, system states are first estimated using the Kalman filter and then using these estimated states LQR controller is designed [11].

Optimal control has been around for a long time. One of the methods of optimal design, that are presently at hand, which is continuously getting recognition is the LQG. The optimality concept is closely related to the control design of multiple inputs multiple outputs (MIMO) systems. Optimum controllers, otherwise best possible, are called optimal controllers. Optimal controllers usually give rise to controllers that stabilize MIMO plants based on some performance criteria. This way, a design process that is automated is provided by optimal control solutions. So, the designer will only have to decide the performance criteria.

Even though LQG is a controller that is gaining popularity in recent years, it is hardly covered in the literature for the ball-on-sphere system. But adaptive feedback linearization control has been covered for the system. Based on the recent review of the literature, the researcher did not come across an LQG design, that uses the control method approach used in this research, for the ball-on-sphere system.

To address the above-mentioned issue (lack of LQG controller design for the ball-on-sphere system in the literature), the research designed an optimal LQG controller for the system of ball-on-sphere. Specifically, the research derived the dynamical equations and state space of the system, designed the controller, and analyzed its performance.

The paper's aim is designing of an optimal LQG for the ball-on-sphere system.

The major research objectives relevant to the research aim are

- To investigate the dynamics and derive the mathematical model of the system.

- To linearize the system due to its nonlinear nature.

- To derive the state space representation of the system.

- To design an optimal LQG controller by finding the relevant matrices using the trial and error method.

- To analyze the performance of the controller. 
No particular method can be argued to be the best because every method has its advantages and disadvantages. The limitation of this research is that the controller is linear while the system is nonlinear. Therefore, the linearized system gives a minimum workable system.

Broadly speaking, the research aims to achieve an at least $80 \%$ settling time less than unregulated settling time of the ball's angles with respect to both $x$ and $y$ directions, zero overshoot of the ball's angles, and all angles should settle to zero or remain very close to zero.

\section{Literature Review}

A system of an inverted pendulum (IP) is stabilized in [12] using state-based observer optimal LQG control. Based on the separation principle, first, all system states were assumed not to be fully measurable, and measurement and process noises corrupted the system thereby designing the Kalman filter. Then, a state feedback controller was designed by obtaining the control law.

The designed controller was then simulated. From the results obtained, it was observed that measurement and process noises were eliminated and the controller stabilized the system.

Robustness and closed-loop performance are determined by the choice of $\mathbf{Q}$ and $\mathbf{R}$ matrices in the design of optimal LQG control. It is a well-known fact that a system like an electro-pneumatic actuator is difficult to control. Based on the existing literature, [13] developed the LQG synthesis methodology and applied it to this system.

Kalman filter and LQR were designed in [14] by taking, system performance and measurement and random system noises, into consideration. Therefore, the controller is an LQG. Then, internal model control (IMC) controller was also integrated. The simulation results showed that parameter variation influence can be dealt with by this method. Good stability and strong robustness were also observed. The proposed strategy is suitable for engineering applications and is easy to regulate.

Ball's position on a beam was controlled using an LQR designed and simulated in [15]. The control was done by changing the beam's angular position. Observer-based state feedback, since not all the system states are measurable, was applied. The LQR scheme modelling as well as simulation to control the system were done with the help of MATLAB/SIMULINK. The LQR scheme effectively stabilized the system with the sensor noises' effect in simulation. LQG and LQR performances were comparatively analyzed.

[16] presented basic theory related to LQG and LQR controllers' design process. Tests were carried out for performance evaluation of a digital autopilot using an LQG/LQR controller. The tests considered stability requirements and different performance criteria given by the regulatory agencies. An aircraft simulation model was used to test the robustness of the design.

By considering the friction effect, [7] outlined the ball-on-sphere system's analysis model. The bond graph technique was used to model the system. Based on the technique, proper causality assignment of dissipating elements, various subsystems, transformer elements, junction structures, storage elements, and energy exchange that make the system up were located and modelled.

\section{System's Mathematical Modelling and Dynamics}

One of the important parts of the control system design process is the modelling or representing the system. To design and analyze controllers, understanding the physical system is a requirement. An accurate dynamical system's representation or model helps us in this understanding. Differential equations describe the dynamical systems' behaviour. Deriving these equations, from the laws of physics, is among the first design process stages. There are different methods of deriving these equations. The Newtonian and Lagrangian methods are the two most commonly used methods for mechanical systems. Ideally, these methods should give the same results. But the system's nature determines which of the methods is simpler for that particular system. Because the dynamic equations, of the ball-onsphere system, are highly nonlinear and coupled, it is difficult to find the significant dynamics of the system. Therefore, dynamical equations are derived by adopting the system's representation or model [17]-[18]. System's parameters: $R$ and $r$, which are the sphere and ball radii respectively, $m$ is the ball mass, $I_{B}$ and $I_{b}$, which are the sphere and ball inertia moments respectively, $\theta_{y}, \beta_{y}$, which are the ball and sphere angles with respect to $y-$ direction respectively, $\theta_{x}, \beta_{x}$, which are the ball and sphere angles with respect to $x$-direction respectively [19]. The system's schema in 2$\mathrm{D}$ is shown in Fig. 1. while Fig. 2. shows the ball-on-sphere system in 3-D. 


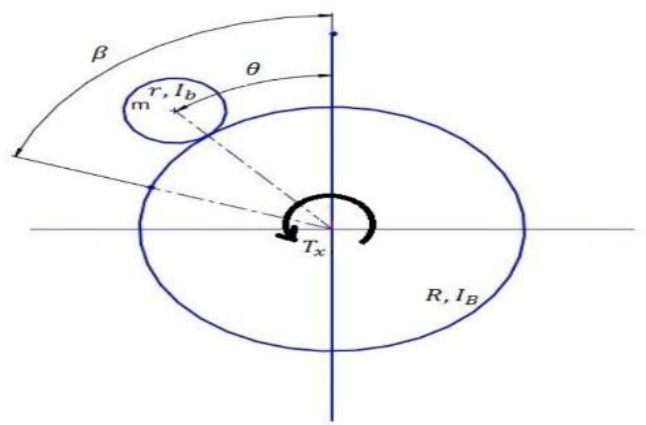

Fig. 1. System's schema in 2-D [20].

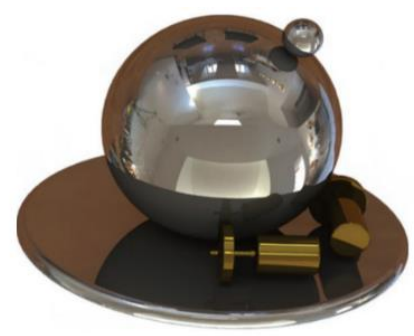

Fig. 2. System of ball-on-sphere [19].

The Lagrangian method is used in deriving the equations as follows [21]:

$$
L=T-V
$$

where $V, T$, and $L$ are the potential and kinetic energy, and the Lagrangian respectively.

$$
\frac{\partial}{\partial t}\left(\frac{\partial L}{\partial \dot{q}}\right)-\frac{\partial L}{\partial q}=Q_{i}, \quad i=1,2,3,4
$$

where $q$ and $Q$ are the generalized coordinate and torque, respectively.

$$
\begin{gathered}
\left\{\begin{array}{c}
Q_{1}=0 \\
Q_{2}=T_{x} \\
Q_{3}=0 \\
Q_{4}=T_{y}
\end{array}\right. \\
q=\left[\begin{array}{llll}
\theta_{x} & \beta_{x} & \theta_{y} & \beta_{y}
\end{array}\right]^{\mathrm{T}}
\end{gathered}
$$

With these assumptions [20]

- The ball is rolling, with no slipping, on the sphere.

- The sphere and ball are in contact always

- No friction effect

and from the linearized model with respect to the equilibrium point, the system can be decoupled into the following two independent subsystems

$$
\begin{gathered}
\left((R+r) m+I_{b} \frac{R+r}{r^{2}}\right) \ddot{\theta}_{x}+\left(-I_{b} \frac{R}{r^{2}}\right) \ddot{\beta}_{x}-m g \sin \left(\theta_{x}\right)=0 \\
\left(-I_{b} \frac{R(R+r)}{r^{2}}\right) \ddot{\theta}_{x}+\left(I_{B}+I_{b} \frac{R^{2}}{r^{2}}\right) \ddot{\beta}_{x}=T_{x} \\
\left((R+r) m+I_{b} \frac{R+r}{r^{2}}\right) \ddot{\theta}_{y}+\left(-I_{b} \frac{R}{r^{2}}\right) \ddot{\beta}_{y}-m g \sin \left(\theta_{y}\right)=0
\end{gathered}
$$




$$
\left(-I_{b} \frac{R(R+r)}{r^{2}}\right) \ddot{\theta}_{y}+\left(I_{B}+I_{b} \frac{R^{2}}{r^{2}}\right) \ddot{\beta}_{y}=T_{y}
$$

Thus, near the equilibrium point, the system can be separated into 2 independent systems of ball and wheel. These subsystems are independently treated and controlled by a controller individually in the $x$ and $y$ directions [20].

The system has 8 state variables because each of the 4 differential equations, $(5)-(8)$, is of the second order. State variables are:

$$
\left\{\begin{array}{l}
x_{1}=\theta_{x} \\
x_{2}=\dot{\theta}_{x} \\
x_{3}=\beta_{x} \\
x_{4}=\dot{\beta}_{x} \\
x_{5}=\theta_{y} \\
x_{6}=\dot{\theta}_{y} \\
x_{7}=\beta_{y} \\
x_{8}=\dot{\beta}_{y}
\end{array}\right.
$$

Taking the derivative of equation (9) and using equation (4) we then get the following equation

$$
\left\{\begin{array}{l}
\dot{x}_{1}=x_{2} \\
\dot{x}_{2}=\ddot{q}_{1} \\
\dot{x}_{3}=x_{4} \\
\dot{x}_{4}=\ddot{q}_{2} \\
\dot{x}_{5}=x_{6} \\
\dot{x}_{6}=\ddot{q}_{3} \\
\dot{x}_{7}=x_{8} \\
\dot{x}_{8}=\ddot{q}_{4}
\end{array}\right.
$$

Assuming $\theta_{x}, \beta_{x}, \theta_{y}$ and $\beta_{y}, T_{x}$ and $T_{y}$ to be the system's outputs and inputs respectively. By arbitrarily naming the state variables, we get

$$
\left\{\begin{array}{l}
x_{1}=\theta_{x} \\
x_{2}=\beta_{x} \\
x_{3}=\theta_{y} \\
x_{4}=\beta_{y} \\
x_{5}=\dot{\theta}_{x} \\
x_{6}=\dot{\beta}_{x} \\
x_{7}=\dot{\theta}_{y} \\
x_{8}=\dot{\beta}_{y}
\end{array}\right.
$$

We get, from equation (11), the first 4 state equations as;

$$
\begin{aligned}
& \dot{x}_{1}=x_{5} \\
& \dot{x}_{2}=x_{6} \\
& \dot{x}_{3}=x_{7} \\
& \dot{x}_{4}=x_{8}
\end{aligned}
$$

By taking $\theta$ and $\beta$ at the equilibrium point to be small, the system can be linearized [22]. This is to $\operatorname{say,} \sin (\theta)=$ $\theta, \cos (\theta)=1, \sin (\beta)=\beta$, and $\cos (\beta)=1$.

Then substituting these assumptions into equations (5) - (8) gives;

$$
\begin{gathered}
a \ddot{\theta}_{x}+b \ddot{\beta}_{x}-m g \theta_{x}=0 \\
c \ddot{\theta}_{x}+d \ddot{\beta}_{x}=T_{x}
\end{gathered}
$$




$$
\begin{gathered}
a \ddot{\theta}_{y}+b \ddot{\beta}_{y}-m g \theta_{y}=0 \\
c \ddot{\theta}_{y}+b \ddot{\beta}_{y}=T_{y}
\end{gathered}
$$

where

$$
\left\{a=\left((R+r) m+I_{b} \frac{R+r}{r^{2}}\right), b=\left(-I_{b} \frac{R}{r^{2}}\right), c=\left(-I_{b} \frac{R(R+r)}{r^{2}}\right), d=\left(I_{B}+I_{b} \frac{R^{2}}{r^{2}}\right)\right.
$$

The 4 remaining state equations can then be expressed as

$$
\begin{gathered}
\dot{x}_{5}=v x_{1}-w T_{x} \\
\dot{x}_{6}=z x_{1}-e T_{x} \\
\dot{x}_{7}=v x_{3}-w T_{y} \\
\dot{x}_{8}=z x_{3}-e T_{y}
\end{gathered}
$$

where

$$
\left\{v=\frac{d m g}{a d-b c}, \quad w=\frac{b}{a d-b c}, \quad z=\frac{c m g}{b c-a d}, \quad e=\frac{a}{b c-a d}\right.
$$

In matrix form,

The output equations are;

$$
\left[\begin{array}{l}
\dot{x}_{1} \\
\dot{x}_{2} \\
\dot{x}_{3} \\
\dot{x}_{4} \\
\dot{x}_{5} \\
\dot{x}_{6} \\
\dot{x}_{7} \\
\dot{x}_{8}
\end{array}\right]=\left[\begin{array}{llllllll}
0 & 0 & 0 & 0 & 1 & 0 & 0 & 0 \\
0 & 0 & 0 & 0 & 0 & 1 & 0 & 0 \\
0 & 0 & 0 & 0 & 0 & 0 & 1 & 0 \\
0 & 0 & 0 & 0 & 0 & 0 & 0 & 1 \\
v & 0 & 0 & 0 & 0 & 0 & 0 & 0 \\
z & 0 & 0 & 0 & 0 & 0 & 0 & 0 \\
0 & 0 & v & 0 & 0 & 0 & 0 & 0 \\
0 & 0 & z & 0 & 0 & 0 & 0 & 0
\end{array}\right]\left[\begin{array}{l}
x_{1} \\
x_{2} \\
x_{3} \\
x_{4} \\
x_{5} \\
x_{6} \\
x_{7} \\
x_{8}
\end{array}\right]+\left[\begin{array}{cc}
0 & 0 \\
0 & 0 \\
0 & 0 \\
0 & 0 \\
-w & 0 \\
-e & 0 \\
0 & -w \\
0 & -e
\end{array}\right]\left[\begin{array}{l}
T_{x} \\
T_{y}
\end{array}\right]
$$

$$
\begin{gathered}
\theta_{x}=x_{1} \\
\beta_{x}=x_{2} \\
\theta_{y}=x_{3} \\
\beta_{y}=x_{4}
\end{gathered}
$$

In matrix form,

$$
\left[\begin{array}{l}
\theta_{x} \\
\beta_{x} \\
\theta_{y} \\
\beta_{y}
\end{array}\right]=\left[\begin{array}{llllllll}
1 & 0 & 0 & 0 & 0 & 0 & 0 & 0 \\
0 & 1 & 0 & 0 & 0 & 0 & 0 & 0 \\
0 & 0 & 1 & 0 & 0 & 0 & 0 & 0 \\
0 & 0 & 0 & 1 & 0 & 0 & 0 & 0
\end{array}\right]\left[\begin{array}{l}
x_{1} \\
x_{2} \\
x_{3} \\
x_{4} \\
x_{5} \\
x_{6} \\
x_{7} \\
x_{8}
\end{array}\right]+\left[\begin{array}{ll}
0 & 0 \\
0 & 0 \\
0 & 0 \\
0 & 0
\end{array}\right]\left[\begin{array}{l}
T_{x} \\
T_{y}
\end{array}\right]
$$

Then the coefficient matrices are; 


$$
\begin{aligned}
& \mathbf{A}=\left[\begin{array}{llllllll}
0 & 0 & 0 & 0 & 1 & 0 & 0 & 0 \\
0 & 0 & 0 & 0 & 0 & 1 & 0 & 0 \\
0 & 0 & 0 & 0 & 0 & 0 & 1 & 0 \\
0 & 0 & 0 & 0 & 0 & 0 & 0 & 1 \\
v & 0 & 0 & 0 & 0 & 0 & 0 & 0 \\
Z & 0 & 0 & 0 & 0 & 0 & 0 & 0 \\
0 & 0 & v & 0 & 0 & 0 & 0 & 0 \\
0 & 0 & Z & 0 & 0 & 0 & 0 & 0
\end{array}\right] \\
& \mathbf{B}=\left[\begin{array}{cc}
0 & 0 \\
0 & 0 \\
0 & 0 \\
0 & 0 \\
-w & 0 \\
-e & 0 \\
0 & -w \\
0 & -e
\end{array}\right] \\
& \mathbf{C}=\left[\begin{array}{llllllll}
1 & 0 & 0 & 0 & 0 & 0 & 0 & 0 \\
0 & 1 & 0 & 0 & 0 & 0 & 0 & 0 \\
0 & 0 & 1 & 0 & 0 & 0 & 0 & 0 \\
0 & 0 & 0 & 1 & 0 & 0 & 0 & 0
\end{array}\right] \\
& \mathbf{D}=\left[\begin{array}{ll}
0 & 0 \\
0 & 0 \\
0 & 0 \\
0 & 0
\end{array}\right]
\end{aligned}
$$

\section{LQG Design}

Two stages of design are involved in the LQG controller design

- LQ Controller (LQR) design

- LQ Estimator (Kalman filter) design

After designing them separately, then they are combined to form the LQG controller.

The purpose of LQG design is to control systems and the design follows the separation principle.

Given the system's dynamics

$$
\begin{aligned}
\dot{\mathbf{x}} & =\mathbf{A x}+\mathbf{B u} \\
\mathbf{y} & =\mathbf{C x}+\mathbf{D u}
\end{aligned}
$$

Using a cost function

$$
J=\int_{0}^{\infty}\left(\mathbf{x}^{\mathrm{T}} \mathbf{Q} \mathbf{x}+\mathbf{u}^{\mathrm{T}} \mathbf{R} \mathbf{u}\right) \mathrm{d} t
$$

the optimal gain matrix is found by the LQR control.

Choosing $\mathbf{R}$ and $\mathbf{Q}$ as diagonal matrices is the conventional and practicable method of choice.

The lowest cost is provided by the gain matrix when the LQR problem is solved. Then,

$$
\mathbf{u}=-\mathbf{K x}
$$

where $\mathbf{K}$

$$
\mathbf{K}=\mathbf{R}^{-1} \mathbf{B}^{\mathrm{T}} \mathbf{P}
$$

$\mathbf{P}$ can be found by solving the Riccati equation

$$
\mathbf{A}^{\mathrm{T}} \mathbf{P}+\mathbf{P A}-\mathbf{P B R}^{-1} \mathbf{B}^{\mathrm{T}} \mathbf{P}+\mathbf{Q}=0
$$


Adjusting $\mathbf{R}$, penalizes the actuator effort while adjusting $\mathbf{Q}$ penalizes bad performance. Input vector judges actuator effort while state vector judges performance.

An optimal prediction-correction estimation is implemented by the Kalman filter, which is basically a mathematical set of equations. It is optimal because the estimated error covariance is minimized when some assumed conditions are satisfied [23].

Fig. 3. shows the Kalman filter used as an optimal observer while Fig. 4. shows the LQG regulator.

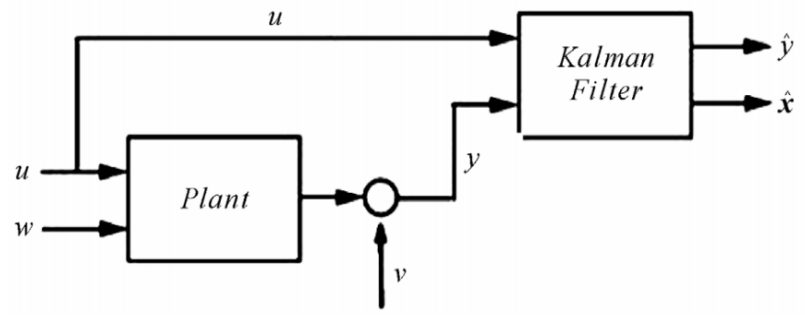

Fig. 3. Kalman filter used as an optimal observer [24].

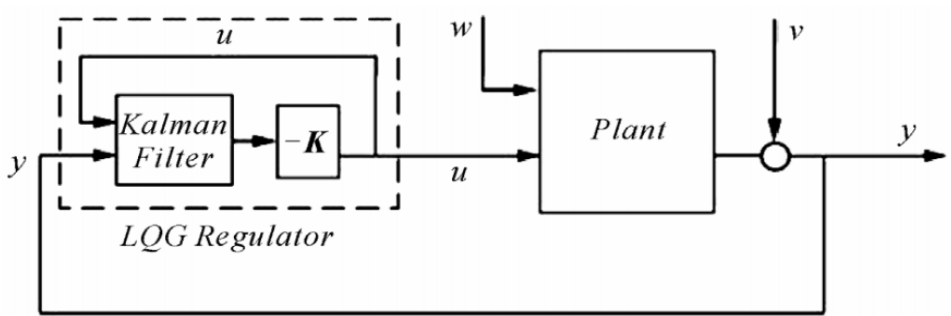

Fig. 4. LQG regulator [24].

A collection of recursive equations plus the system's discrete model is what the Kalman filter's general form usually contains. $\mathbf{P}$, system covariance matrix, and $\mathbf{L}$, Kalman gain matrix are continuously updated by these recursive equations [25].

From a collection of recursive equations, $\mathbf{L}$ can be obtained. These recursive equations start from some starting covariance matrix $\mathbf{P}(k / k)$

$$
\begin{gathered}
\mathbf{P}(k+1 / k)=\mathbf{A}(T) \mathbf{P}(k / k) \mathbf{A}^{\mathrm{T}}(T)+\mathbf{C}_{\mathrm{d}}(T) \mathbf{Q}_{\mathrm{e}} \mathbf{C}_{\mathrm{d}}^{\mathrm{T}}(T) \\
\mathbf{L}(k+1)=\mathbf{P}(k+1 / k) \mathbf{C}^{\mathrm{T}}(T)\left\{\mathbf{C}(T) \mathbf{P}(k+1 / k) \mathbf{C}^{\mathrm{T}}(T)+\mathbf{R}_{\mathrm{e}}\right\}^{-1} \\
\mathbf{P}(k+1 / k+1)=\{\mathbf{I}-\mathbf{L}(k+1) \mathbf{C}(T)\} \mathbf{P}(k+1 / k)
\end{gathered}
$$

The process goes on by putting the covariance $\mathbf{P}(k+1 / k+1)$, (42), back into equation (40) as $\mathbf{P}(k / k)$ till $\mathbf{L}(k+1)$ goes to a steady value.

Here, $\mathbf{C}_{\mathrm{d}}(T), \mathbf{R}_{\mathrm{e}}$, and $\mathbf{Q}_{\mathrm{e}}$ are the disturbance transition, measurement noise covariance, and disturbance noise covariance matrices, respectively.

Let $\mathbf{P}(k / k)=\mathbf{P}_{1}, \mathbf{P}(k+1 / k+1)=\mathbf{P}_{3}$, and $\mathbf{P}(k+1 / k)=\mathbf{P}_{2}$, then equations (40) - (42) become

$$
\begin{gathered}
\mathbf{P}_{2}=\mathbf{A} \mathbf{P}_{1} \mathbf{A}^{\mathrm{T}}+\mathbf{C}_{\mathrm{d}} \mathbf{Q}_{\mathrm{e}} \mathbf{C}_{\mathrm{d}}^{\mathrm{T}} \\
\mathbf{L}=\mathbf{P}_{2} \mathbf{C}^{\mathrm{T}}\left\{\mathbf{C} \mathbf{P}_{2} \mathbf{C}^{\mathrm{T}}+\mathbf{R}_{\mathrm{e}}\right\}^{-1} \\
\mathbf{P}_{3}=\{\mathbf{I}-\mathbf{L} \mathbf{C}\} \mathbf{P}_{2}
\end{gathered}
$$

\section{Results and Analysis}

The regulation was done based on the following system performance:

- The ball's angle with respect to both $x$ and $y$ directions should have at least $80 \%$ settling time less than its unregulated settling time. 
- The ball's angles should have zero overshoot.

- All angles should settle to zero or remain very close to zero.

The system's physical parameters were given arbitrary values for the sake of controlling the system. These are presented in Table 1.

Table 1. Desired system's physical parameters [26].

\begin{tabular}{|c|c|c|}
\hline Parameter & Value & Units \\
\hline Mass of the ball $(m)$ & 0.06000 & $\mathrm{~kg}$ \\
\hline The radius of the ball $(r)$ & 0.01250 & $\mathrm{~m}$ \\
\hline The radius of the sphere $(R)$ & 0.15000 & $\mathrm{~m}$ \\
\hline Ball's moment of inertia $\left(I_{b}\right)$ & $3.75 \times 10^{-6}$ & $\mathrm{~kg} \cdot \mathrm{m}^{2}$ \\
\hline Sphere's moment of inertia $\left(I_{B}\right)$ & 0.99 & $\mathrm{~kg} \cdot \mathrm{m}^{2}$ \\
\hline Gravitational acceleration $(g)$ & 9.81 & $\mathrm{~m} / \mathrm{s}^{2}$ \\
\hline
\end{tabular}

The system, system states, were assigned desired initial conditions, and this assignment is done arbitrarily. These are presented in Table 2.

Table 2. System's desired initial conditions [26].

\begin{tabular}{|c|c|c|}
\hline Initial parameter & Value & Units \\
\hline$\theta_{x 0}$ & 0.07 & $\mathrm{rad}$ \\
\hline$\beta_{x 0}$ & 0 & $\mathrm{rad}$ \\
\hline$\theta_{y 0}$ & 0.07 & $\mathrm{rad}$ \\
\hline$\beta_{y 0}$ & 0 & $\mathrm{rad}$ \\
\hline$\dot{\theta}_{x 0}$ & 0.02 & $\mathrm{rad} / \mathrm{s}$ \\
\hline$\dot{\beta}_{x 0}$ & 0 & $\mathrm{rad} / \mathrm{s}$ \\
\hline$\dot{\theta}_{y 0}$ & 0.05 & $\mathrm{rad} / \mathrm{s}$ \\
\hline$\dot{\beta}_{y 0}$ & 0 & $\mathrm{rad} / \mathrm{s}$ \\
\hline
\end{tabular}

Based on the system's physical parameters presented in Table 1., the coefficient matrices have the following numerical values:

$$
\begin{gathered}
\mathbf{A}=\left[\begin{array}{ccccccccc}
0 & 0 & 0 & 0 & 1 & 0 & 0 & 0 \\
0 & & 0 & 0 & 0 & 0 & 1 & 0 & 0 \\
0 & & 0 & 0 & 0 & 0 & 0 & 1 & 0 \\
0 & 0 & 0 & 0 & 0 & 0 & 0 & 1 \\
43.1276 & 0 & 0 & 0 & 0 & 0 & 0 & 0 \\
0.0255 & 0 & 0 & 0 & 0 & 0 & 0 & 0 \\
0 & 43.1276 & 0 & 0 & 0 & 0 & 0 & 0 \\
0 & 0.0255 & 0 & 0 & 0 & 0 & 0 & 0
\end{array}\right] \quad \mathbf{B}=\left[\begin{array}{ccc}
0 & 0 \\
0 & 0 \\
0 & 0 \\
0 & 0 \\
0.2663 & 0 \\
1.0097 & 0 \\
0 & 0.2663 \\
0 & 1.0097
\end{array}\right] \\
\mathbf{C}=\left[\begin{array}{llllllll}
1 & 0 & 0 & 0 & 0 & 0 & 0 & 0 \\
0 & 1 & 0 & 0 & 0 & 0 & 0 & 0 \\
0 & 0 & 1 & 0 & 0 & 0 & 0 & 0 \\
0 & 0 & 0 & 1 & 0 & 0 & 0 & 0
\end{array}\right]
\end{gathered}
$$

Using the desired initial conditions in Table 2., for the unregulated control of the system, the inputs to the system are $58.5 \mathrm{Joule} / \mathrm{rad}$ and $58.4 \mathrm{Joule} / \mathrm{rad}$ maximum and $-13.90 \mathrm{Joule} / \mathrm{rad}$ and $-14.80 \mathrm{Joule} / \mathrm{rad}$ minimum in the $x$ and $y$ directions, respectively, while the settling time in the $x$-direction is $6.65 \mathrm{sec}$ and $6.70 \mathrm{sec}$ in the $y$-direction. It can be noticed that the settling time and maximum input (torque) are almost the same for both directions even though the angular velocities are different. It was observed that for small angular velocities, settling time and maximum input value are independent of angular velocity. The input responses are shown in Fig. 5. and Fig. 6. for $x$ and $y$ directions, respectively. 


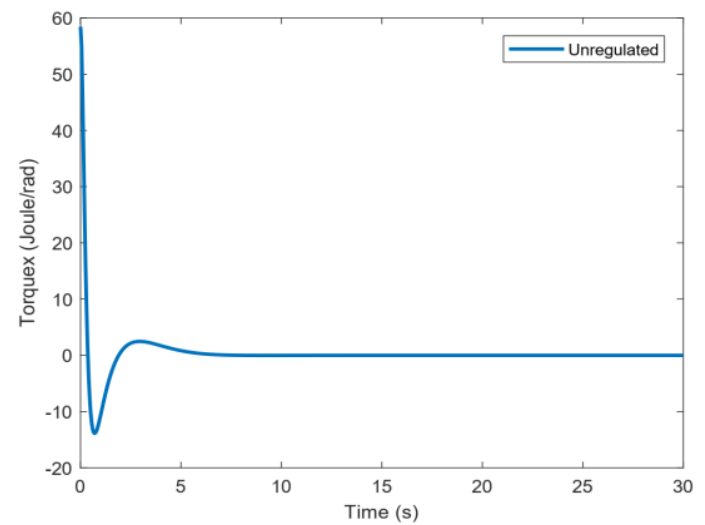

Fig. 5. Unregulated input response in the $x$ - direction, $T_{x}$.

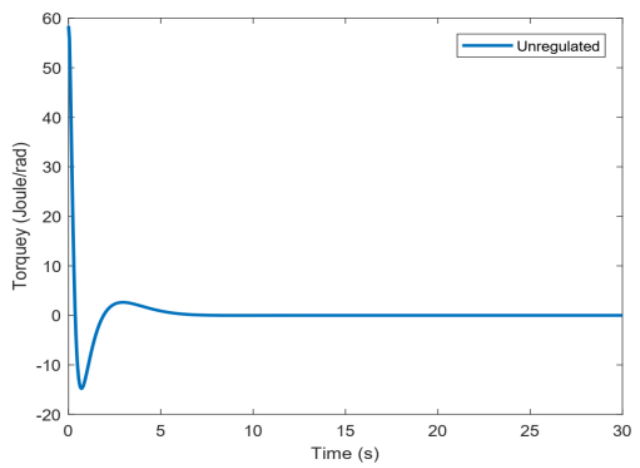

Fig. 6. Unregulated input response in the $y$ - direction, $T_{y}$.

From Fig. 7. and Fig. 8., the settling time is $6.80 \mathrm{sec}$ for the ball's angle with respect to the $x$ - direction while it is $6.75 \mathrm{sec}$ with respect to the $y$-direction. With different angular velocities, it can be noticed that the settling time is almost the same for both directions.

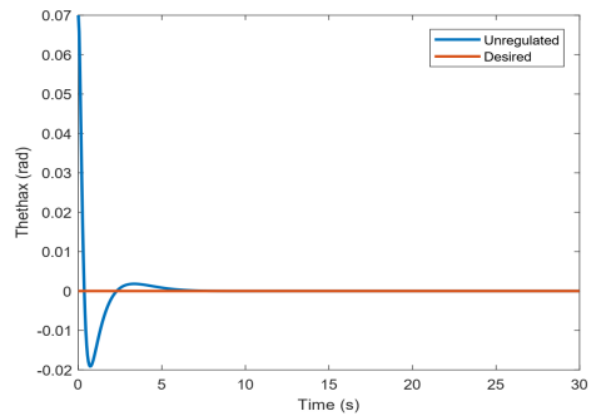

Fig. 7. Unregulated output response in the $x$ - direction, $\theta_{x}$.

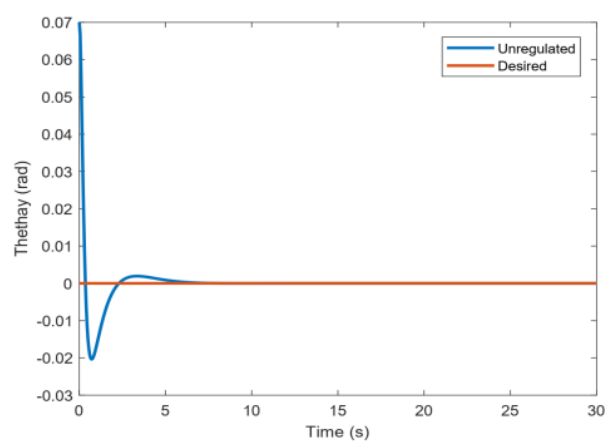

Fig. 8. Unregulated output response in the $y$ - direction, $\theta_{y}$. 
The sphere's angle response settles faster in the $y$ - direction than in the $x$-direction with 6.90 sec settling time in the $y$-direction and $7.10 \mathrm{sec}$ in the $x$-direction, Fig. 9. and Fig. 10., even though angular velocity is higher in the $y$ - direction. This shows that settling time and angular velocity are inversely proportional to the sphere's angle.

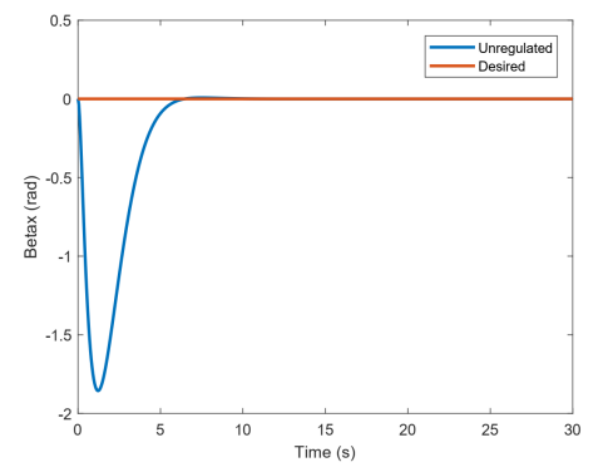

Fig. 9. Unregulated output response in the $x$ - direction, $\beta_{x}$.

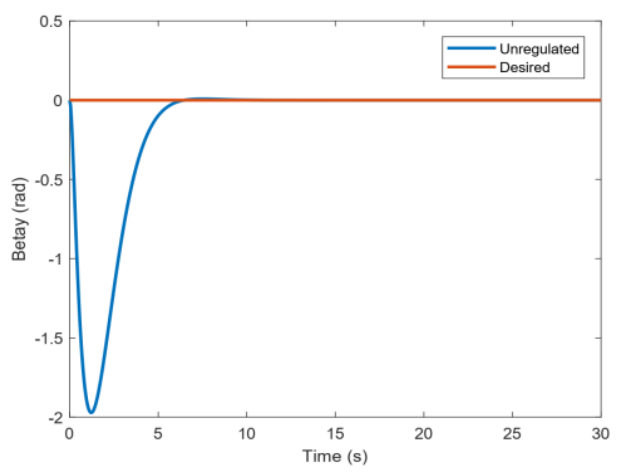

Fig. 10. Unregulated output response in the $y-$ direction, $\beta_{y}$.

The matrices that give these unregulated responses in Fig. 5. through Fig. 10. are as below.

$$
\begin{gathered}
\mathbf{Q}=\left[\begin{array}{cccccccc}
1 & 0 & 0 & 0 & 0 & 0 & 0 & 0 \\
0 & 1 & 0 & 0 & 0 & 0 & 0 & 0 \\
0 & 0 & 1 & 0 & 0 & 0 & 0 & 0 \\
0 & 0 & 0 & 1 & 0 & 0 & 0 & 0 \\
0 & 0 & 0 & 0 & 1 & 0 & 0 & 0 \\
0 & 0 & 0 & 0 & 0 & 1 & 0 & 0 \\
0 & 0 & 0 & 0 & 0 & 0 & 1 & 0 \\
0 & 0 & 0 & 0 & 0 & 0 & 0 & 1
\end{array}\right] \quad \mathbf{R}=\left[\begin{array}{cc}
1 & 0 \\
0 & 1
\end{array}\right] \quad \mathbf{E}=\left[\begin{array}{c}
-6.7017+0.0000 \mathrm{i} \\
-6.7017+0.0000 \mathrm{i} \\
-6.4354+0.0000 \mathrm{i} \\
-6.4354+0.0000 \mathrm{i} \\
-0.8715+0.0000 \mathrm{i} \\
-0.8715-0.0000 \mathrm{i} \\
-0.8715+0.0000 \mathrm{i} \\
-0.8715-0.0000 \mathrm{i}
\end{array}\right] \\
\mathbf{K}=\left[\begin{array}{ccccccccc}
417.4791 & -1.0000 & -0.0000 & 0.0000 & 63.5795 & -2.0312 & -0.0000 & 0.0000 \\
-0.0000 & 0.0000 & 417.4791 & -1.0000 & -0.0000 & 0.0000 & 63.5795 & -2.0312
\end{array}\right] \\
\mathbf{P}=10^{4}\left[\begin{array}{ccccccccc}
1.3272 & -0.0064 & -0.0000 & 0.0000 & 0.2021 & -0.0119 & -0.0000 & 0.0000 \\
-0.0064 & 0.0002 & 0.0000 & -0.0000 & -0.0010 & 0.0002 & 0.0000 & -0.0000 \\
-0.0000 & 0.0000 & 1.3272 & -0.0064 & -0.0000 & 0.0000 & 0.2021 & -0.0119 \\
0.0000 & -0.0000 & -0.0064 & 0.0002 & 0.0000 & -0.0000 & -0.0010 & 0.0002 \\
0.2021 & -0.0010 & -0.0000 & 0.0000 & 0.0308 & -0.0018 & -0.0000 & 0.0000 \\
-0.0119 & 0.0002 & 0.0000 & -0.0000 & -0.0018 & 0.0003 & 0.0000 & -0.0000 \\
-0.0000 & 0.0000 & 0.2021 & -0.0010 & -0.0000 & 0.0000 & 0.0308 & -0.0018 \\
0.0000 & -0.0000 & -0.0119 & 0.0002 & 0.0000 & -0.0000 & -0.0018 & 0.0003
\end{array}\right]
\end{gathered}
$$




$$
\begin{aligned}
& \mathbf{A}(T)=10^{3}\left[\begin{array}{cccccccc}
0.3557 & 0 & 0 & 0 & 0.0542 & 0 & 0 & 0 \\
0.0002 & 0.0010 & 0 & 0 & 0.0000 & 0.0010 & 0 & 0 \\
0 & 0 & 0.3557 & 0 & 0 & 0 & 0.0542 & 0 \\
0 & 0 & 0.0002 & 0.0010 & 0 & 0 & 0.0000 & 0.0010 \\
2.3358 & 0 & 0 & 0 & 0.3557 & 0 & 0 & 0 \\
0.0014 & 0 & 0 & 0 & 0.0002 & 0.0010 & 0 & 0 \\
0 & 0 & 2.3358 & 0 & 0 & 0 & 0.3557 & 0 \\
0 & 0 & 0.0014 & 0 & 0 & 0 & 0.0002 & 0.0010
\end{array}\right] \\
& \mathbf{B}(T)=\left[\begin{array}{cc}
2.1900 & 0 \\
0.5061 & 0 \\
0 & 2.1900 \\
0 & 0.5061 \\
14.4224 & 0 \\
1.0181 & 0 \\
0 & 14.4224 \\
0 & 1.0181
\end{array}\right] \quad \mathbf{C}_{\mathrm{d}}=\left[\begin{array}{cccccccc}
1 & 0 & 0 & 0 & 0 & 0 & 0 & 0 \\
0 & 1 & 0 & 0 & 0 & 0 & 0 & 0 \\
0 & 0 & 1 & 0 & 0 & 0 & 0 & 0 \\
0 & 0 & 0 & 1 & 0 & 0 & 0 & 0 \\
0 & 0 & 0 & 0 & 1 & 0 & 0 & 0 \\
0 & 0 & 0 & 0 & 0 & 1 & 0 & 0 \\
0 & 0 & 0 & 0 & 0 & 0 & 1 & 0 \\
0 & 0 & 0 & 0 & 0 & 0 & 0 & 1
\end{array}\right] \quad \mathbf{R}_{\mathrm{e}}=\left[\begin{array}{cccc}
1 & 0 & 0 & 0 \\
0 & 1 & 0 & 0 \\
0 & 0 & 1 & 0 \\
0 & 0 & 0 & 1
\end{array}\right] \\
& \mathbf{Q}_{\mathrm{e}}=\left[\begin{array}{llllllll}
1 & 0 & 0 & 0 & 0 & 0 & 0 & 0 \\
0 & 1 & 0 & 0 & 0 & 0 & 0 & 0 \\
0 & 0 & 1 & 0 & 0 & 0 & 0 & 0 \\
0 & 0 & 0 & 1 & 0 & 0 & 0 & 0 \\
0 & 0 & 0 & 0 & 1 & 0 & 0 & 0 \\
0 & 0 & 0 & 0 & 0 & 1 & 0 & 0 \\
0 & 0 & 0 & 0 & 0 & 0 & 1 & 0 \\
0 & 0 & 0 & 0 & 0 & 0 & 0 & 1
\end{array}\right] \quad \mathbf{L}=\left[\begin{array}{cccc}
1.0000 & 0.0001 & 0 & 0 \\
0.0001 & 0.7647 & 0 & 0 \\
0 & 0 & 1.0000 & 0.0001 \\
0 & 0 & 0.0001 & 0.7647 \\
6.5667 & 0.0010 & 0 & 0 \\
0.0037 & 0.3529 & 0 & 0 \\
0 & 0 & 6.5667 & 0.0010 \\
0 & 0 & 0.0037 & 0.3529
\end{array}\right] \\
& \mathbf{P}_{3}=\left[\begin{array}{cccccccc}
1.0000 & 0.0001 & 0 & 0 & 6.5667 & 0.0037 & 0 & 0 \\
0.0001 & 0.7647 & 0 & 0 & 0.0010 & 0.3529 & 0 & 0 \\
0 & 0 & 1.0000 & 0.0001 & 0 & 0 & 6.5667 & 0.0037 \\
0 & 0 & 0.0001 & 0.7647 & 0 & 0 & 0.0010 & 0.3529 \\
6.5667 & 0.0010 & 0 & 0 & 44.1184 & 0.0244 & 0 & 0 \\
0.0037 & 0.3529 & 0 & 0 & 0.0244 & 1.4706 & 0 & 0 \\
0 & 0 & 6.5667 & 0.0010 & 0 & 0 & 44.1184 & 0.0244 \\
0 & 0 & 0.0037 & 0.3529 & 0 & 0 & 0.0244 & 1.4706
\end{array}\right]
\end{aligned}
$$

Here $\mathbf{A}(T)$ is the discrete-time transition matrix, $\mathbf{B}(T)$ is the discrete-time control matrix, $\mathbf{C}_{\mathrm{d}}$ is the disturbance matrix, $\mathbf{R}_{\mathbf{e}}$ is the measurement noise covariance matrix, $\mathbf{Q}_{\mathrm{e}}$ is the disturbance noise covariance matrix, $\mathbf{L}$ is the Kalman gain matrix, and $\mathbf{P}_{3}$ is the estimation error covariance matrix after 0 iterations.

Regulating the system/controller matrices to get the desired system performance, two of the many inputs that meet the desired performance are found to have $48.1 \mathrm{Joule} / \mathrm{rad}$ and $50.0 \mathrm{Joule} / \mathrm{rad}$ in $x$ and $y$ directions, respectively, with a settling time of $1.20 \mathrm{sec}$ for input in the $x$-direction and $1.10 \mathrm{sec}$ for input in the $y$-direction. These responses are shown in Fig. 11. and Fig. 12. For the regulated responses the relationship between input and settling time, as well as input and angular velocity, cannot be deduced since many inputs can satisfy the desired system performance.

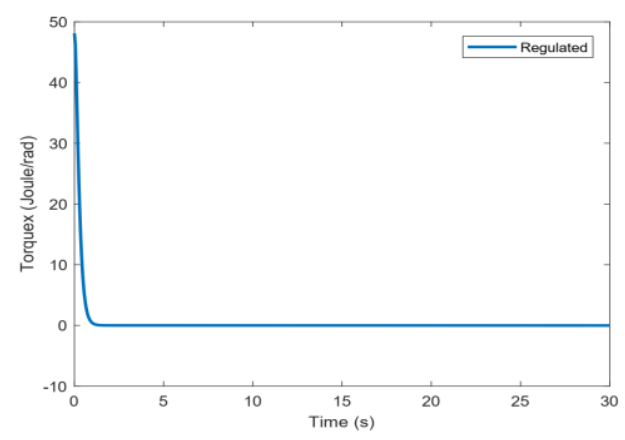

Fig. 11. Regulated input response in the $x$-direction, $T_{x}$. 


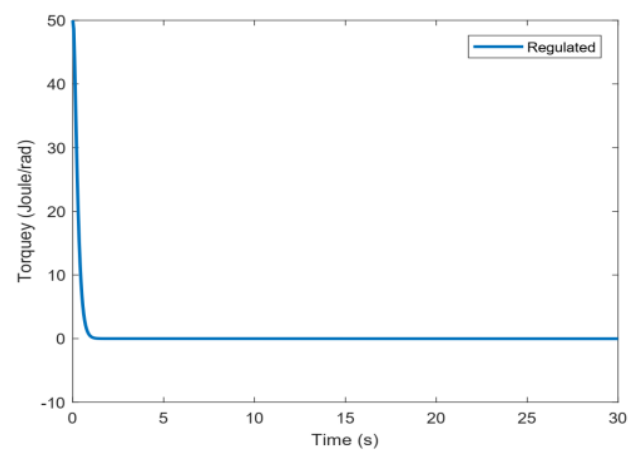

Fig. 12. Regulated input response in the $y$ - direction, $T_{y}$.

For the regulated system, the output responses have a settling time of $1.20 \mathrm{sec}$ and $1.15 \mathrm{sec}$ for the ball's angle with respect to $x$ and $y$ directions, respectively. The $1.20 \mathrm{sec}$ settling time of $\theta_{x}$ is $17.65 \%$ of its unregulated settling time (Fig. 7.) which is $6.80 \mathrm{sec}$. This means that $\theta_{x}$ has a settling time $82.35 \%$ less than its unregulated settling time. While $\theta_{y}$ has a settling time $82.96 \%$ less than its unregulated settling time (Fig. 8.). Efforts were made to get a settling time of less than $1.00 \mathrm{sec}$ for the regulated responses but unfortunately, such value could not be realized. The responses are shown in Fig. 13. and Fig. 14.

It is also observed that all states play role in regulating the system to desired system performance.

Fig. 15. and Fig. 16. show the regulated output responses $\beta_{x}$ and $\beta_{y}$.

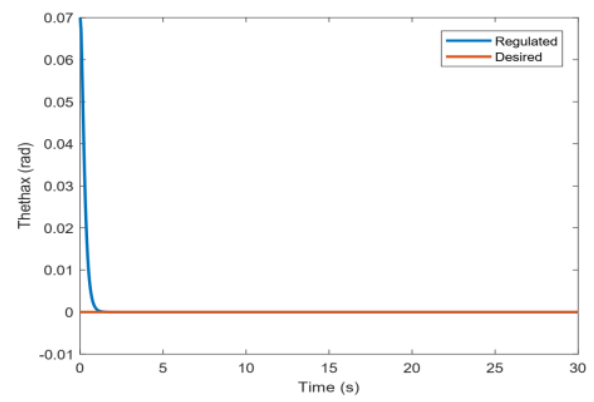

Fig. 13. Regulated output response in the $x$ - direction, $\theta_{x}$.

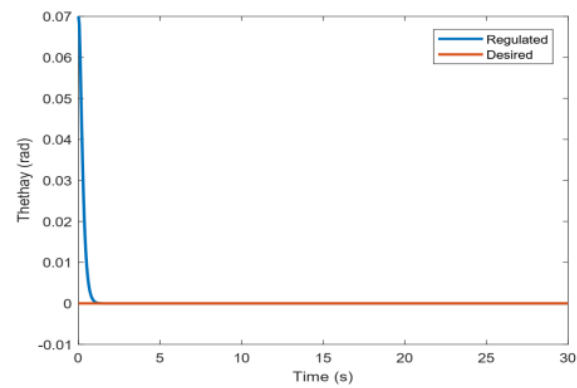

Fig. 14. Regulated output response in the $y-$ direction, $\theta_{y}$.

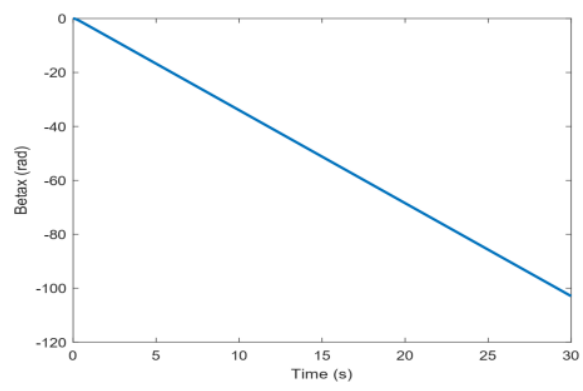

Fig. 15. Regulated output response in the $x$-direction, $\beta_{x}$. 


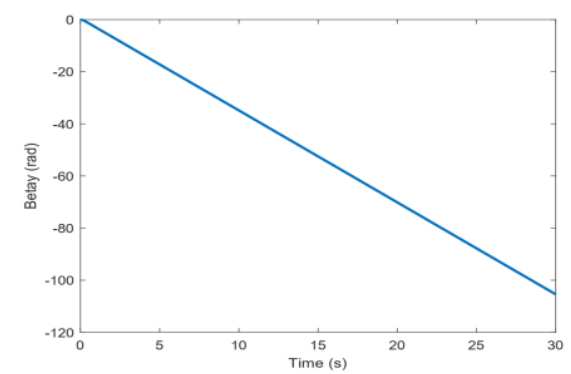

Fig. 16. Regulated output response in the $y-$ direction, $\beta_{y}$.

The regulated system matrices are as below.

It is observed that all states play role in regulating the system to desired system performance.

$$
\begin{aligned}
& \mathbf{Q}=10^{-7}\left[\begin{array}{cccccccc}
1 & 0 & 0 & 0 & 0 & 0 & 0 & 0 \\
0 & 1 & 0 & 0 & 0 & 0 & 0 & 0 \\
0 & 0 & 1 & 0 & 0 & 0 & 0 & 0 \\
0 & 0 & 0 & 1 & 0 & 0 & 0 & 0 \\
0 & 0 & 0 & 0 & 1 & 0 & 0 & 0 \\
0 & 0 & 0 & 0 & 0 & 1 & 0 & 0 \\
0 & 0 & 0 & 0 & 0 & 0 & 1 & 0 \\
0 & 0 & 0 & 0 & 0 & 0 & 0 & 1
\end{array}\right] \quad \mathbf{R}=10^{13}\left[\begin{array}{ll}
1 & 0 \\
0 & 1
\end{array}\right] \quad \mathbf{E}=\left[\begin{array}{l}
-6.5672+0.0000 \mathrm{i} \\
-6.5672+0.0000 \mathrm{i} \\
-6.5672+0.0000 \mathrm{i} \\
-6.5672+0.0000 \mathrm{i} \\
-0.0000+0.0000 \mathrm{i} \\
-0.0000-0.0000 \mathrm{i} \\
-0.0000+0.0000 \mathrm{i} \\
-0.0000-0.0000 \mathrm{i}
\end{array}\right] \\
& \mathbf{K}=\left[\begin{array}{cccccccc}
300.8385 & -0.0000 & 42.7703 & -0.0000 & 45.8095 & -0.0000 & 6.5128 & -0.0000 \\
42.7703 & -0.0000 & 314.3527 & -0.0000 & 6.5127 & -0.0000 & 47.8674 & -0.0000
\end{array}\right] \\
& \mathbf{P}=10^{16}\left[\begin{array}{cccccccc}
7.4190 & -0.0000 & 1.0548 & -0.0000 & 1.1297 & -0.0000 & 0.1606 & -0.0000 \\
-0.0000 & 0.0000 & -0.0000 & -0.0000 & -0.0000 & 0.0000 & -0.0000 & -0.0000 \\
1.0548 & -0.0000 & 7.7523 & -0.0000 & 0.1606 & -0.0000 & 1.1805 & -0.0000 \\
-0.0000 & -0.0000 & -0.0000 & 0.0000 & -0.0000 & -0.0000 & -0.0000 & 0.0000 \\
1.1297 & -0.0000 & 0.1606 & -0.0000 & 0.1720 & -0.0000 & 0.0245 & -0.0000 \\
-0.0000 & 0.0000 & -0.0000 & -0.0000 & -0.0000 & 0.0000 & -0.0000 & -0.0000 \\
0.1606 & -0.0000 & 1.1805 & -0.0000 & 0.0245 & -0.0000 & 0.1798 & -0.0000 \\
-0.0000 & -0.0000 & -0.0000 & 0.0000 & -0.0000 & -0.0000 & -0.0000 & 0.0000
\end{array}\right] \\
& \mathbf{A}(T)=10^{3}\left[\begin{array}{cccccccc}
0.3557 & 0 & 0 & 0 & 0.0542 & 0 & 0 & 0 \\
0.0002 & 0.0010 & 0 & 0 & 0.0000 & 0.0010 & 0 & 0 \\
0 & 0 & 0.3557 & 0 & 0 & 0 & 0.0542 & 0 \\
0 & 0 & 0.0002 & 0.0010 & 0 & 0 & 0.0000 & 0.0010 \\
2.3358 & 0 & 0 & 0 & 0.3557 & 0 & 0 & 0 \\
0.0014 & 0 & 0 & 0 & 0.0002 & 0.0010 & 0 & 0 \\
0 & 0 & 2.3358 & 0 & 0 & 0 & 0.3557 & 0 \\
0 & 0 & 0.0014 & 0 & 0 & 0 & 0.0002 & 0.0010
\end{array}\right] \\
& \mathbf{B}(T)=\left[\begin{array}{cc}
2.1900 & 0 \\
0.5061 & 0 \\
0 & 2.1900 \\
0 & 0.5061 \\
14.4224 & 0 \\
1.0181 & 0 \\
0 & 14.4224 \\
0 & 1.0181
\end{array}\right] \quad \mathbf{C}_{\mathrm{d}}=\left[\begin{array}{cccccccc}
0.1 & 0 & 0 & 0 & 0 & 0 & 0 & 0 \\
0 & 0.1 & 0 & 0 & 0 & 0 & 0 & 0 \\
0 & 0 & 0.1 & 0 & 0 & 0 & 0 & 0 \\
0 & 0 & 0 & 0.1 & 0 & 0 & 0 & 0 \\
0 & 0 & 0 & 0 & 0.1 & 0 & 0 & 0 \\
0 & 0 & 0 & 0 & 0 & 0.1 & 0 & 0 \\
0 & 0 & 0 & 0 & 0 & 0 & 0.1 & 0 \\
0 & 0 & 0 & 0 & 0 & 0 & 0 & 0.1
\end{array}\right]
\end{aligned}
$$




$$
\mathbf{R}_{\mathrm{e}}=10^{-3}\left[\begin{array}{llll}
1 & 0 & 0 & 0 \\
0 & 1 & 0 & 0 \\
0 & 0 & 1 & 0 \\
0 & 0 & 0 & 1
\end{array}\right]
$$

\subsection{Summary}

$$
\mathbf{Q}_{\mathrm{e}}=\left[\begin{array}{cccccccc}
0.1 & 0 & 0 & 0 & 0 & 0 & 0 & 0 \\
0 & 0.1 & 0 & 0 & 0 & 0 & 0 & 0 \\
0 & 0 & 0.1 & 0 & 0 & 0 & 0 & 0 \\
0 & 0 & 0 & 0.1 & 0 & 0 & 0 & 0 \\
0 & 0 & 0 & 0 & 0.1 & 0 & 0 & 0 \\
0 & 0 & 0 & 0 & 0 & 0.1 & 0 & 0 \\
0 & 0 & 0 & 0 & 0 & 0 & 0.1 & 0 \\
0 & 0 & 0 & 0 & 0 & 0 & 0 & 0.1
\end{array}\right]
$$

The controller results and performance can be summarized as below:

- Larger input gives a longer settling time of input and output responses.

- Minimizing the total control energy leads to maximizing the total transient energy.

- Input is independent of angular velocity and settling time.

- For small angular velocities, the ball's angles' settling time is independent of angular velocity and input.

- Settling time and angular velocity are inversely proportional to the sphere's angle.

- The relationship between input and settling time, as well as input and angular velocity, cannot be deduced since many inputs can satisfy the desired system performance.

- $\theta_{x}$ has a settling time $82.35 \%$ less than its unregulated settling time while that of $\theta_{y}$ is $82.96 \%$ less than its unregulated settling time.

- All states play role in regulating the system to the desired system performance.

- $\quad$ Recursive iterations lead (not generally) to unstable input.

Table 3. gives a numerical summary of the controller results and performance.

Table 3. Summary of the controller results and performance.

\begin{tabular}{|c|c|c|c|c|c|c|}
\hline Response & Type & Direction & $\begin{array}{c}\text { Settling } \\
\text { Time }\end{array}$ & Maximum Value & Minimum Value & Regulation \\
\hline$T_{x}$ & Input & $x-$ direction & $6.65 \mathrm{sec}$ & $58.5 \mathrm{Joule} / \mathrm{rad}$ & $-13.90 \mathrm{Joule} / \mathrm{rad}$ & Unregulated \\
\hline$T_{y}$ & Input & $y-$ direction & $6.70 \mathrm{sec}$ & $58.4 \mathrm{Joule} / \mathrm{rad}$ & $-14.80 \mathrm{Joule} / \mathrm{rad}$ & Unregulated \\
\hline$\theta_{x}$ & Output & $x-$ direction & $6.80 \mathrm{sec}$ & $0.070 \mathrm{rad}$ & $-0.01908 \mathrm{rad}$ & Unregulated \\
\hline$\theta_{y}$ & Output & $y-$ direction & $6.75 \mathrm{sec}$ & $0.070 \mathrm{rad}$ & $-0.02031 \mathrm{rad}$ & Unregulated \\
\hline$\beta_{x}$ & Output & $x-$ direction & $7.10 \mathrm{sec}$ & $0 \mathrm{rad}$ & $-1.85600 \mathrm{rad}$ & Unregulated \\
\hline$\beta_{y}$ & Output & $y-$ direction & $6.90 \mathrm{sec}$ & $0 \mathrm{rad}$ & $-1.97200 \mathrm{rad}$ & Unregulated \\
\hline$T_{x}$ & Input & $x-$ direction & $1.20 \mathrm{sec}$ & $48.1 \mathrm{Joule} / \mathrm{rad}$ & $0 \mathrm{Joule} / \mathrm{rad}$ & Regulated \\
\hline$T_{y}$ & Input & $y-$ direction & $1.10 \mathrm{sec}$ & $50.0 \mathrm{Joule} / \mathrm{rad}$ & $0 \mathrm{Joule} / \mathrm{rad}$ & Regulated \\
\hline$\theta_{x}$ & Output & $x-$ direction & $1.20 \mathrm{sec}$ & $0.070 \mathrm{rad}$ & $0 \mathrm{rad}$ & Regulated \\
\hline$\theta_{y}$ & Output & $y-$ direction & $1.15 \mathrm{sec}$ & $0.070 \mathrm{rad}$ & $0 \mathrm{rad}$ & Regulated \\
\hline
\end{tabular}




\section{Conclusion}

LQG is a controller that is gaining popularity among the optimal controllers currently available but unfortunately, it is rarely covered in the literature for an important system like ball-on-sphere. So, the main aim of this research work was to design an LQG for the ball-on-sphere system.

This aim was achieved by executing the goals as follows: first investigating the system's dynamics and deriving the mathematical model of the system using the Lagrangian method by considering some assumptions. Then, the system was linearized (due to its nonlinear nature) and a state-space representation of the system was derived. Using the statespace model of the system, an optimal LQG controller was then designed and implemented using MATLAB, first without regulation, and then the controller was regulated (through manipulation of matrices by trial and error method) to get the desired system performance. Finally, the controller's performance was analyzed.

It was found out that the controller could not be regulated for the output (ball's angles) responses to have a settling time of less than $1.00 \mathrm{sec}$. but it was able to satisfy the desired system performance requirement set by the research work. Another finding was that the controller minimizes the total control energy (while maximizing the total transient energy).

With this research, the identified gap in the literature has been bridged and future work can use this research as a basis.

\section{References}

[1] N. S. Nise, CONTROL SYSTEMS ENGINEERING, 6th Edition. California State Polytechnic University, Pomona: John Wiley \& Sons, Inc., 2011.

[2] B. Xu, 'A Comparative Study of PID and LQR Control Strategies Applied to Inverted Pendulum Systems', Master of Engineering, M.Eng, University of Guelph, Ontario, Canada, 2019.

[3] E. Zakeri, S. A. Moezi, and Y. Bazargan-Lari, 'Control of a Ball on Sphere System with Adaptive Feedback Linearization method for regulation purpose', Majlesi J. Mechatron. Eng., vol. 2, no. 3, pp. 23-27, Sep. 2013.

[4] Y.-H. Chang, W.-S. Chan, and C.-W. Chang, 'T-S Fuzzy Model-Based Adaptive Dynamic Surface Control for Ball and Beam System', IEEE Trans. Ind. Electron., vol. 60, no. 6, pp. 2251-2263, Jun. 2013, doi: 10.1109/TIE.2012.2192891.

[5] M.-T. Ho, Y.-W. Tu, and H.-S. Lin, 'Controlling a ball and wheel system using full-state-feedback linearization [Focus on Education]', IEEE Control Syst., vol. 29, no. 5, pp. 93-101, Oct. 2009, doi: 10.1109/MCS.2009.934085.

[6] A. Buschhaus and S. Schmal, 'Robolab Reutlingen University', https://vvl.reutlingen-university.de/homepage/en/index.html. https://vvl.reutlingen-university.de/homepage/en/index.html\#demos/ballOnBall/main (accessed Aug. 26, 2020).

[7] A. D. Usman, A. M. Yusuf, A. Umar, and A. Daniel, 'Structual analysis of ball-on-sphere system using bond graph technique', in 2017 IEEE 3rd International Conference on Electro-Technology for National Development (NIGERCON), Owerri, Nov. 2017, pp. 519-524, doi: 10.1109/NIGERCON.2017.8281921.

[8] M.-T. Ho, Y. Rizal, and W.-S. Cheng, 'Stabilization of a Vision-Based Ball-On-Sphere System', in 2013 IEEE International Conference on Control Applications (CCA), Part of 2013 IEEE Multi-Conference on Systems and Control, Hyderabad, India, Aug. 2013, pp. 929-934, doi: 10.1109/CCA.2013.6662870.

[9] A. Umar, Z. Haruna, U. Musa, S. A. Mohammed, and M. O. Muyideen, 'Graphical User Interface (GUI) for Position and Trajectory Tracking Control of the Ball and Plate System Using H-Infinity Controller’, doi: 10.20370/YHAS-N460.

[10] H. Jafari, A. Rahimpour, and M. Pourrahim, 'Linear Quadratic Gaussian Control for ball and plate system', in 2012 international conference on computer, control, education and management, Dubai, United Arab Emirates, Jul. 2012, pp. 1-7.

[11] W. Favoreell and B. D. Moor, 'Model-free subspace-based LQG-design', in Proceedings of the American Control Conference (ACC), San Diego, California, Jun. 1999, pp. 3372-3376, doi: 10.1109/ACC.1999.782390.

[12] R. Banerjee and A. Pal, 'Stabilization of Inverted Pendulum on Cart Based on LQG Optimal Control', in 2018 International Conference on Circuits and Systems in Digital Enterprise Technology (ICCSDET), Kottayam, India, Dec. 2018, pp. 1-4, doi: 10.1109/ICCSDET.2018.8821243.

[13] A. Besancon-Voda, G. Filardi, D. Rey, and A. Franco, 'LQG optimal control strategies for an electro pneumatic actuator', in 2001 European Control Conference (ECC), Porto, Sep. 2001, pp. 2670-2675, doi: 10.23919/ECC.2001.7076333.

[14] Q. Jin, S. Ren, and Ling Quan, 'LQG optimum controller design and simulation base on inter model control theory', in 2009 IEEE International Conference on Intelligent Computing and Intelligent Systems, Shanghai, China, Nov. 2009, pp. 62-65, doi: 10.1109/ICICISYS.2009.5358234.

[15] R. Soni and Sathans, 'Optimal control of a ball and beam system through LQR and LQG', in 2018 2nd International Conference on Inventive Systems and Control (ICISC), Coimbatore, Jan. 2018, pp. 179-184, doi: 10.1109/ICISC.2018.8399060.

[16] J. Vlk and P. Chudy, 'General aviation digital autopilot design based on LQR/LQG control strategy', in 2017 IEEE/AIAA 36th Digital Avionics Systems Conference (DASC), St. Petersburg, FL, Sep. 2017, pp. 1-9, doi: 10.1109/DASC.2017.8102058.

[17] M. Ho, Y. Tu, and H. Lin, 'Controlling a ball and wheel system using full-state-feedback linearization [Focus on Education]', Control Syst. IEEE, vol. 29, pp. 93-101, Nov. 2009, doi: 10.1109/MCS.2009.934085.

[18] M. Moarref, M. Saadat, and G. Vossoughi, 'Mechatronic design and position control of a novel ball and plate system', in 2008 16th Mediterranean Conference on Control and Automation, Ajaccio, France, Jun. 2008, pp. 1071-1076, doi: 10.1109/MED.2008.4602212. 
[19] S. A. Moezi, E. Zakeri, Y. Bazargan-Lari, and M. Khalghollah, 'Fuzzy Logic Control of a Ball on Sphere System', Adv. Fuzzy Syst., vol. 2014, pp. 1-6, 2014, doi: 10.1155/2014/291430.

[20] S.-Y. Liu, Y. Rizal, and M.-T. Ho, 'Stabilization of a Ball and Sphere System Using Feedback Linearization and Sliding Mode Control', in 2011 8th Asian Control Conference (ASCC), May 2011, p. 6.

[21] E. Zakeri, A. Ghahramani, and S. Moezi, 'Adaptive Feedback Linearization Control of a Ball on Sphere System', in International Conference on Mechanical Engineering and Advanced Technology, ICMEAT 2012, Isfahan, Iran, Oct. 2012, pp. $1-5$.

[22] A. Tewari, Modern control design with MATLAB and SIMULINK. New York: John Wiley, 2002.

[23] G. Welch and G. Bishop, 'An Introduction to the Kalman Filter'. 2006.

[24] R. Eide, P. M. Egelid, A. Stamsø, and H. R. Karimi, 'LQG Control Design for Balancing an Inverted Pendulum Mobile Robot', Intell. Control Autom., vol. 02, no. 02, pp. 160-166, 2011, doi: 10.4236/ica.2011.22019.

[25] R. S. Burns, Advanced control engineering. Oxford, Boston: Butterworth-Heinemann, 2001.

[26] U. Mohammed, S. U. Hussein, M. Usman, and S. Thomas, 'Design of an Optimal Linear Quadratic Regulator (LQR) Controller for the Ball-On-Sphere System', International Journal of Engineering and Manufacturing(IJEM), vol. 10, no. 3, pp. 56-70, Jun. 2020, doi: 10.5815/ijem.2020.03.05.

\section{Author's Profile}

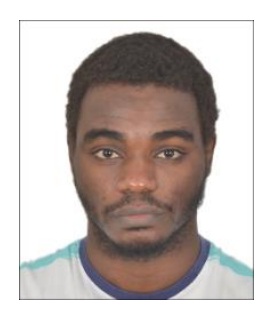

Usman Mohammed is a student in the Department of Electrical and Electronics Engineering, Nile University of Nigeria, Abuja, Nigeria. He completed his Bachelor's degree in Physics in 2016 at Fatih University, Istanbul, Turkey, and a Postgraduate Diploma (PGD) in Electrical and Electronics Engineering in 2019 at Nile University of Nigeria, Abuja, Nigeria. Currently, he is pursuing a Master's degree in Electrical and Electronics Engineering at Nile University of Nigeria, Abuja, Nigeria.

How to cite this paper: Usman Mohammed, Tologon Karataev, Omotayo O. Oshiga, Suleiman U. Hussein, " Optimal Controller Design for the System of Ball-on-sphere: The Linear Quadratic Gaussian (LQG) Case ", International Journal of Engineering and Manufacturing (IJEM), Vol.11, No.2, pp. 14-30, 2021. DOI: 10.5815/ijem.2021.02.02 